\title{
Venous stasis orbitopathy: a clinical and echographic study
}

Department of Ophthalmology, University of Aberdeen H R Atta A D Dick

Bascom Palmer Eye Institute, University of Miami, USA

L M Hamed

$S$ F Byrne

E K Gendron

J S Glaser

Correspondence to: Dr H R Atta, Department of Ophthalmology, Medical School, Foresterhill, Aberdeen AB9 2ZD

Aberdeen AB9 2ZD. 23 October 1995

\begin{abstract}
Aims-A variety of acute and chronic orbitopathies may be distinguished by standardised echography. Venous stasis orbitopathy (VSO) often presents with orbital signs when secondary to cavernous sinus and middle cranial fossa disorders. In this study, the aim was to assess whether differentiation between vascular and nonvascular causes of VSO could be made on the basis of clinical and echographic features at the time of presentation.

Methods-This study comprised 37 patients with echographic features of VSO (17 patients with arteriovenous fistulae, confirmed by computed tomography imaging or angiography, and 20 patients with non-vascular diseases). Excluded were patients with orbital mass lesions detected by echography and muscle enlargement due to other causes (for example, orbital myositis). Patients with a suspected mass involving the orbital apex and echographic features of VSO were included. After full neuro-ophthalmic and ocular examination, both orbits were examined to document maximal thickness and reflectivity of four recti muscles and compared with the normal contralateral orbit with standardised A-scan (Kretztechnik 7200MA or Ophthascan) and contact B-scan (Ultrascan or Ophthascan S). Results-Cumulative ocular recti muscle thickness was significantly greater in patients with arteriovenous fistulae compared with the non-fistula group (23.3 (SD 3.7) and $17 \cdot 8$ (2) $\mathrm{mm}, p=0.001)$. Clinically, the presence of a bruit and a uniocular rise in intraocular pressure were significantly greater in the fistula group of patients.
\end{abstract}

Conclusions-Standardised echography is a safe and non-invasive method of diagnosing VSO in patients presenting with signs of proptosis, ophthalmoplegia, and inflammation of the conjunctiva. Furthermore, using these standard techniques the two major causes of VSO (arteriovenous fistulae and compressive mass lesions) could be differentiated.

(Brf Ophthalmol 1996; 80: 129-134)

Since the advent of standardised echography, ${ }^{12}$ the diagnosis and evaluation of many orbital diseases have been greatly enhanced. ${ }^{3-8}$ Although computed tomography (CT) and magnetic resonance imaging (MRI) are very useful in the depiction of orbital, periorbital, and intracranial lesions, standardised echography is, at times, more sensitive in the detection of subtle orbital abnormalities, particularly when modern instrumentation and comprehensive echographic techniques are used. ${ }^{5}$

The clinical diagnosis of acute orbitopathies often requires the assistance of alternative imaging methods. These patients present with several common features, including proptosis, congested and inflamed adnexa and conjunctiva, and external ophthalmoplegia, with or without reduced vision. Standardised echography is a well established method of diagnosing orbital myopathies including Graves' disease, 25910 orbital myositis, ${ }^{11} 12$ and other disorders associated with enlarged extraocular muscles. 23578 These conditions include those entities which obstruct venous outflow, resulting in orbital venous engorgement, enlargement of extraocular muscles, and increased episcleral venous and intraocular pressure. We have termed this constellation of findings 'venous stasis orbitopathy' (VSO); although, in certain high flow fistulae (see below), arterialisation of the superior ophthalmic vein occurs which may result in a paradoxical drainage of blood from an otherwise congested orbit. VSO may be caused by either mass lesions (neoplasm or inflammation) in the middle cranial fossa or at the orbital apex, or by arteriovenous fistulae. The latter group may be further subdivided into carotid-cavernous fistulae and dural-sinus fistulae. ${ }^{13}$

Echography can differentiate fast flow and slow flow fistulae owing to the presence of a fast flow of blood within the superior ophthalmic vein. ${ }^{14}$ The dramatic clinical signs so characteristic of large, fast flow, carotidcavernous fistulae are not present in slow flow indirect dural-sinus fistulae, which show less spectacular clinical signs, rendering the cause of the acute orbitopathy much less obvious. ${ }^{13}$ The diagnoses of other non-vascular causes of VSO (sphenocavernous masses or inflammation) can be difficult on clinical grounds alone. Consequently various imaging modalities, including CT scan, MRI, and orbital echography are often required. ${ }^{15} 16$

We present the clinical and echographic features of 37 patients who presented with a putative diagnosis of VSO and demonstrate the role of standardised echography in the different diagnosis of this group of disorders.

\section{Methods}

PATIENTS

A retrospective study was undertaken to evaluate the clinical, echographic, and neuroimaging findings in 37 patients with VSO who 
presented to the Bascom-Palmer Eye Institute (Miami, FL, USA). The condition of VSO was established on both clinical and echographic findings and orbital diagnosis confirmed with other imaging modalities. Echographically, VSO was diagnosed when unilateral enlargement of multiple extraocular muscles was found in association with one or more of the following: enlargement of orbital veins, thickening of the optic nerve, and swelling of the orbital fat (compared with normal, contralateral orbit). ${ }^{714}$ Patients with orbital mass lesions and those with extraocular muscle enlargement due to other causes (for example, orbital myositis) were excluded. However, patients with a suspected mass involving orbital apex/superior orbital fissure with the echographic features of VSO were included in the study.

\section{ECHOGRAPHIC EVALUATION}

Both orbits of all patients were examined with standardised echography using A-scan (Kretztechnik 7200MA or Ophthascan S) and contact B-scan (Ultrascan or Ophthascan S) to exclude orbital mass lesions and to document maximum thickness and reflectivity of the four recti muscles. ${ }^{7}$ The cumulative thickness of the four recti was calculated and compared with that of the contralateral orbit, as well as with normative values, as previously described by Byrne et al. ${ }^{17}$ Optic nerve diameters were also measured with standardised A-scan in both primary gaze and in $30^{\circ}$ of abduction, to determine whether increased subarachnoid fluid was present. ${ }^{18-20}$ The superior ophthalmic and medial collateral veins ${ }^{21}$ and the orbital fat were also evaluated for evidence of associated engorgement (widening of the echograms obtained from the orbital fat compared with the contralateral normal orbit). A simple nondirectional Doppler ultrasound instrument was also utilised in suspected cases of arteriovenous fistulae to help assess flow dynamics. Statistical analysis was performed using a non-parametric Students' $t$ test.

\section{CLINICAL EVALUATION}

Each patient had undergone a complete evaluation in the Neuro-Ophthalmological service of the Bascom Palmer Eye Institute. Data regarding symptoms, visual acuity, visual field defects, the presence of afferent pupillary defects, and limitation of ocular movements were collected. Motility was graded from $0-4$, where $0=$ normal movement, $1=$ mild restriction, $2=$ moderate restriction, $3=$ severe

Table 1 Clinical diagnoses in 37 patients presenting with venous stasis orbitopathy

\begin{tabular}{ll}
\hline Diagnosis & Number (\%) \\
\hline Arteriovenous fistula & $17(46)$ \\
Indirect (low flow) & $12(32 \cdot 5)$ \\
Direct (high flow) & $5(13 \cdot 5)$ \\
Cavernous sinus/middle fossa disease & $10(27)$ \\
Tolosa-Hunt syndrome & $6(16)$ \\
Orbital apex tumours & $3(8)$ \\
Superior ophthalmic vein thrombosis & $1(3)$ \\
\hline
\end{tabular}

restriction, and $4=$ no movement. Other features evaluated included the presence of cephalic or ocular bruits, applanation tonometry, Hertel exophthalmometry, appearance of conjunctival and fundal vasculature, and the presence of optic disc swelling. Clinical diagnoses were confirmed by CT scan and/or carotid angiography.

\section{Results}

The various clinical diagnoses of the 37 patients are detailed in Table 1. Arteriovenous fistulae accounted for $17 / 37(46 \%)$ of the diagnoses with other, non-fistulae causes of VSO accounting for the remainder. Of these, six cases were consistent with Tolosa-Hunt syndrome/inflammatory orbital apex syndrome.

\section{CLINICAL AND ECHOGRAPHIC FINDINGS}

Clinical examination (Table 2) demonstrated congested conjunctival vessels in all patients and a uniocular rise in intraocular pressure was found in $30 / 37$ patients $(81 \%)$, compared with their fellow normal eye. Proptosis and limitation of ocular movements were respectively noted in $32 / 37(86.5 \%)$ and $29 / 37(78 \%)$ of patients. Impaired visual acuity on presentation was uncommon, with $20 / 37(54 \%)$ of patients presenting with a visual acuity of $6 / 6$, and only two patients presenting with a vision of $6 / 60$ or worse. The absolute uniocular intraocular pressure of the affected eye was greater in patients with vascular fistula compared with non-fistula causes of VSO (mean 27 (SD 5) and 17 (4) $\mathrm{mm} \mathrm{Hg}, \mathrm{p}=0.05$, Fig 1). There was also a difference of IOP between affected and normal eye in both groups, but within the non-vascular group this was not significant (mean 17 (4) and 13.5 (3) $\mathrm{mm} \mathrm{Hg}$ ). There was no significant difference in the degree of proptosis (measured by exophthalmometry), afferent pupillary defect, or venous stasis retinopathy between the two groups of patients. Ocular or cephalic bruits were detected in six patients $(16.7 \%)$, all of whom had VSO as the result of a fistula.

The spectrum of echographic features documented in our group of patients with VSO is detailed in Table 3. All patients were found to have unilateral, diffuse enlargement of extraocular muscles, compared with the contralateral side (Fig 1). Other common echographic features in the 37 patients included optic nerve sheath enlargement (36 patients, 97\%),

Table 2 Clinical manifestations of patients with venous stasis orbitopathy

\begin{tabular}{lc}
\hline Features & Number (\%) \\
\hline Congested conjunctival vessels & $37(100)$ \\
Proptosis & $32(86)$ \\
Uniocular rise in IOP & $30(81)$ \\
Limitation of ocular movement & $29(78)$ \\
Ocular/orbital pain & $18(49)$ \\
Retinal venous congestion & $7(19)$ \\
Bruits & $6(16)$ \\
Visual acuity & $20(54)$ \\
$6 / 6$ & $12(32)$ \\
$6 / 6-6 / 18$ & $3(8)$ \\
$6 / 18-6 / 60$ & $2(5)$ \\
$>6 / 60$ & \\
\hline
\end{tabular}



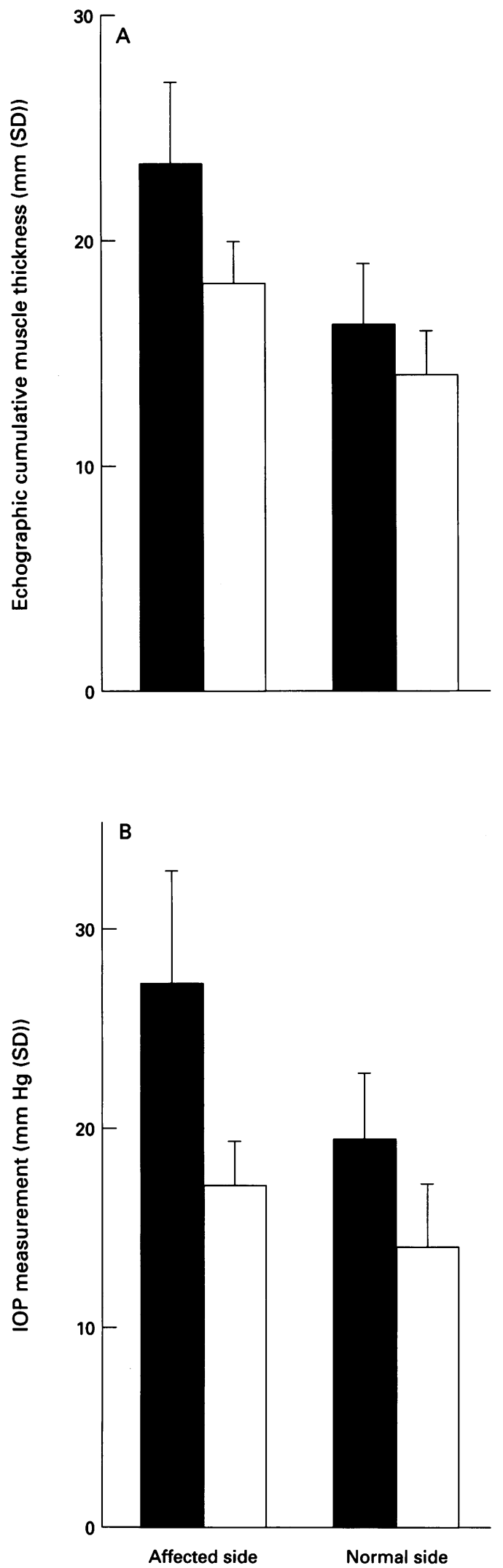

Fistula group Non-fistula group

Figure 1 Comparison of $(A)$ extraocular muscle thickness and (B) intraocular pressure (IOP) in fistula and non-fistula causes of venous stasis orbitopathy.

enlarged orbital veins in 34 patients (92\%), and widening of the orbital fat pattern in 20 patients $(54 \%)$. Within the arteriovenous fistula group, the mean cumulative recti
Table 3 Echographic findings in 37 patients presenting with venous stasis orbitopathy

\begin{tabular}{lc}
\hline Echographic features & Number (\%) \\
\hline Enlarged extraocular muscles & $37(100)$ \\
Distension of optic nerve sheaths & $36(97)$ \\
Enlarged orbital vein* & $34(92)$ \\
Widening of orbital fat pattern & $20(54)$ \\
Widening of superior orbital fissure pattern $\dagger$ & $4(11)$ \\
\hline
\end{tabular}

*Either superior ophthalmic or medial collateral veins.

tWidening of echogram pattern within the superior orbital fissure as a result of greater than normal volume of tissue within the fissure.

thickness was significantly greater than in the non-fistula group $(23(3 \cdot 7)$, and $17 \cdot 8(2) \mathrm{mm}$, $\mathrm{p}=0.001$ ) Although the optic nerve diameter was noted to be increased in VSO, there was no statistical difference in the degree of enlargement between the two groups. The $30^{\circ}$ test for optic nerve sheath fluid was positive in only $6 / 21(29 \%)$ patients where motility was sufficient for the test to be performed; there was no difference between the two groups of patients. There was no statistical difference in any of the other echographic features of VSO (Table 3).

The following two case reports demonstrate the clinical and echographic findings in vascular fistula and non-fistula causes of venous stasis orbitopathy.

\section{Case 1 Dural-sinus fistula}

An 82-year-old women presented with a 1 year history of increasing swelling of the left eyelids, proptosis, and redness. Several months previously, she had complained of diplopia. On examination visual acuity was $6 / 12$ right eye and 6/60 left eye, with a left relative afferent pupillary defect. Left eye ductions were limited, particularly in elevation. The conjunctiva was injected and chemotic with arterialisation of the superficial blood vessels (Fig 2). Intraocular pressure was $18 \mathrm{~mm} \mathrm{Hg}$ right eye and $34 \mathrm{~mm} \mathrm{Hg}$ left eye. There was no audible ocular bruit. Echography demonstrated diffuse marked enlargement of all four recti in the left orbit, with medium reflectivity, and normal muscle insertions (Fig 3), as well as enlargement of the superior ophthalmic vein (Fig 4). The optic nerve was found to be enlarged but the $30^{\circ}$ test could not be performed because of

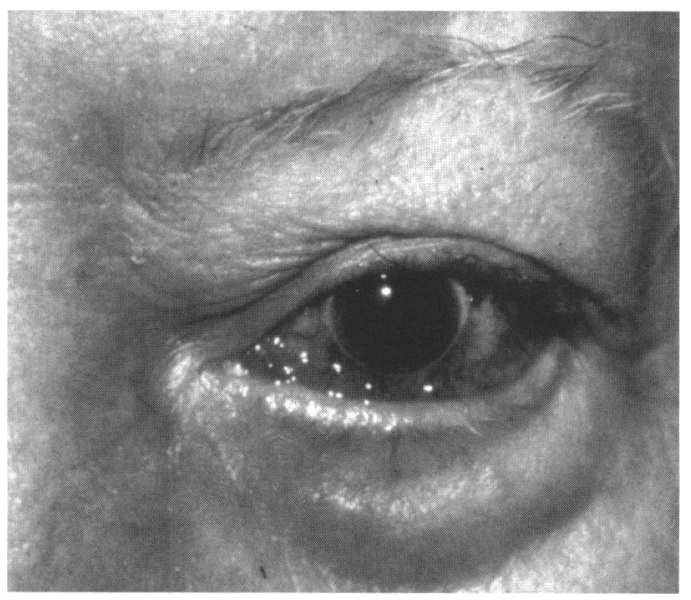

Figure 2 Case 1 shows injection, chemosis, and arterialisation of conjunctival vessels in the left eye. 

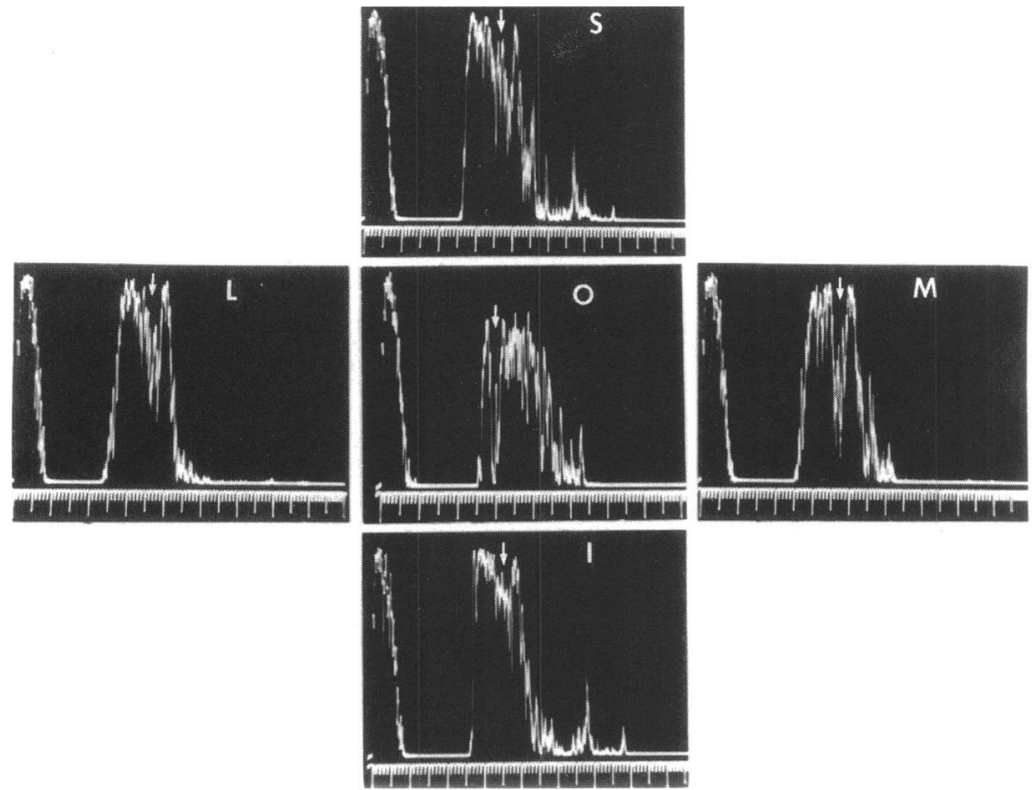

Fig $3 A$
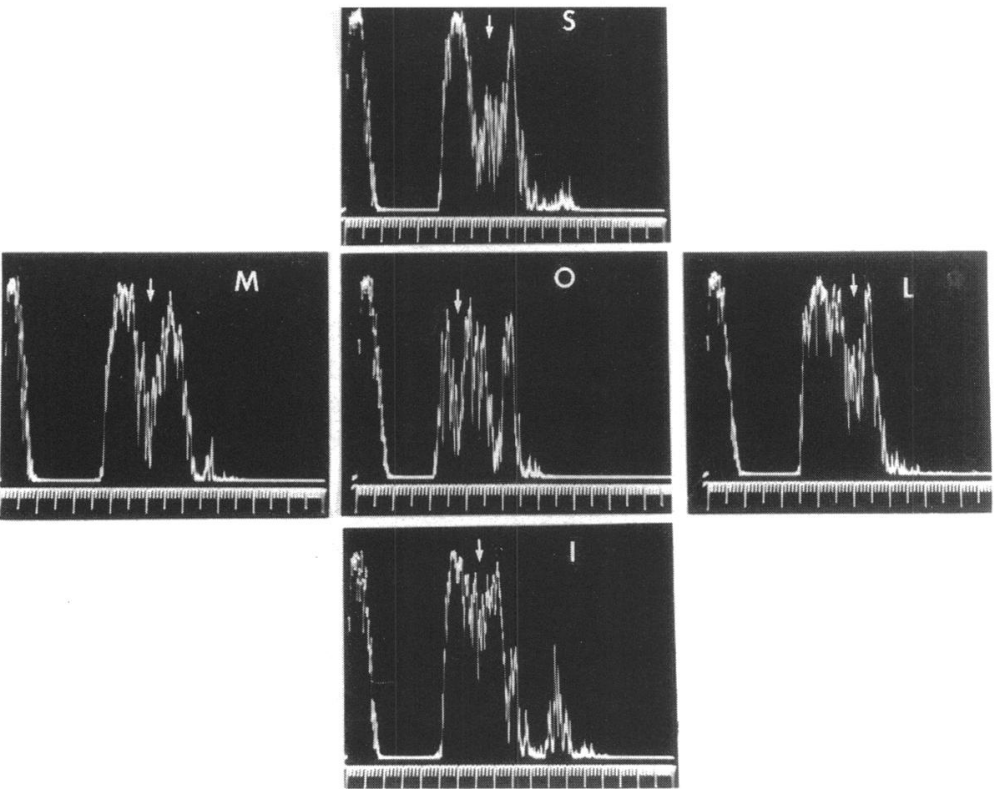

Fig $3 B$

Figure 3 Case 1. Dural sinus fistula. Standardised A-scan echograms of extraocular muscles and optic nerves (arrows) from normal right orbit $(A)$ and abnormal left orbit $(B)$ $S=S$ uperior rectus muscle. $L=$ Lateral rectus muscle. $I=I$ fiferior rectus muscle. $M=M e d i a l$ rectus muscle. $O=$ Optic nerve. $(A) S=4.7 \mathrm{~mm}, L=3.6 \mathrm{~mm}, I=3.7 \mathrm{~mm}, M=3.3 \mathrm{~mm}$, $O=3.1 \mathrm{~mm}$. (B) $S=8.1 \mathrm{~mm}, L=5.2 \mathrm{~mm}, I=9.0 \mathrm{~mm}, M=7.2 \mathrm{~mm}, O=3.6 \mathrm{~mm}$.

limited ocular movements. Cerebral angiography confirmed the presence of a dural-sinus fistula, fed by branches of the external carotid system.

Case 2 Tolosa-Hunt/inflammatory orbital apex syndrome

A 56-year-old woman presented with a painful red left eye and diplopia of a few days' duration. On examination visual acuity was normal but the left conjunctiva was injected and there was marked limitation of ocular movement in all directions of gaze. There was also left ptosis and proptosis, and intraocular pressures were $14 \mathrm{~mm} \mathrm{Hg}$ in both eyes. Echography demonstrated an increase in the thickness of all four recti throughout the length of each muscle in the left orbit, associated at the orbital apex with an increased diameter and low reflectivity of both the orbital fat pattern and superior orbital fissure.

These signs were attributed to a diffuse orbital apex inflammatory mass (Fig 5) associated with a slightly widened superior ophthalmic vein (Fig 6). In addition, the sheaths of the left optic nerve were found to be enlarged. CT scan confirmed enlargement of the superior ophthalmic vein (Fig 7) and demonstrated a fluid level in the maxillary and ethmoidal sinuses suggestive of sinusitis and a non-enhancing, diffuse opacification of the orbital apex. The patient did not respond to antibiotics but enjoyed improvement with systemic steroids.

\section{Discussion}

There is a wide spectrum of differential diagnoses to be considered in patients with an acute orbitopathy that presents with the common signs of proptosis, ophthalmoplegia, chemosis, and inflammation of the lids and conjunctiva, with or without optic nerve involvement. ${ }^{7} 1213$

During a 3 year period we used echography to evaluate venous stasis orbitopathy in 37 patients. The signs and symptoms were caused by either an arteriovenous fistula, a neoplasm, or an inflammation involving the orbital apex-cavernous sinus region (sphenocarvernous syndrome) as confirmed by CT scan and/or angiography. This echographic study was helpful in differentiating these lesions from other acute orbitopathies with similar clinical appearances. In most of our patients with VSO, echography consistently showed the extraocular muscles to be diffusely enlarged with reflectivity generally in the normal range, and sparing the muscle insertions, unlike the low reflectivity and thickened muscle insertions typical of orbital myositis. ${ }^{7} 1112$

In early Graves' orbitopathy, maximum thickening of the extraocular muscles occurs in the muscle bellies, which may also show medium-high reflectivity on A-scan. In the late stages, presumably when the muscles become fibrotic, the reflectivity often increases. ${ }^{2} 347910$ Frequently, the contralateral asymptomatic orbit will also show characteristically enlarged extraocular muscles. Other associated acoustic features of Graves' orbitopathy may include periosteal thickening, and occasionally swelling of the lacrimal gland. In severe cases, marked swelling of the apical portion of the recti can result in the obstruction of venous outflow, compounding the severity of orbitopathy and producing other vascular changes associated with VSO. Consequently, in unilateral Graves' orbitopathy with marked apical muscle enlargement, it may be difficult to differentiate Graves' orbitopathy from other causes of VSO.

When VSO is due to a mass lesion in the orbital apex or cavernous sinus, the offending lesion may not be readily detected on echography, but is demonstrable by CT scan or MRI. In these cases, echography can detect the 


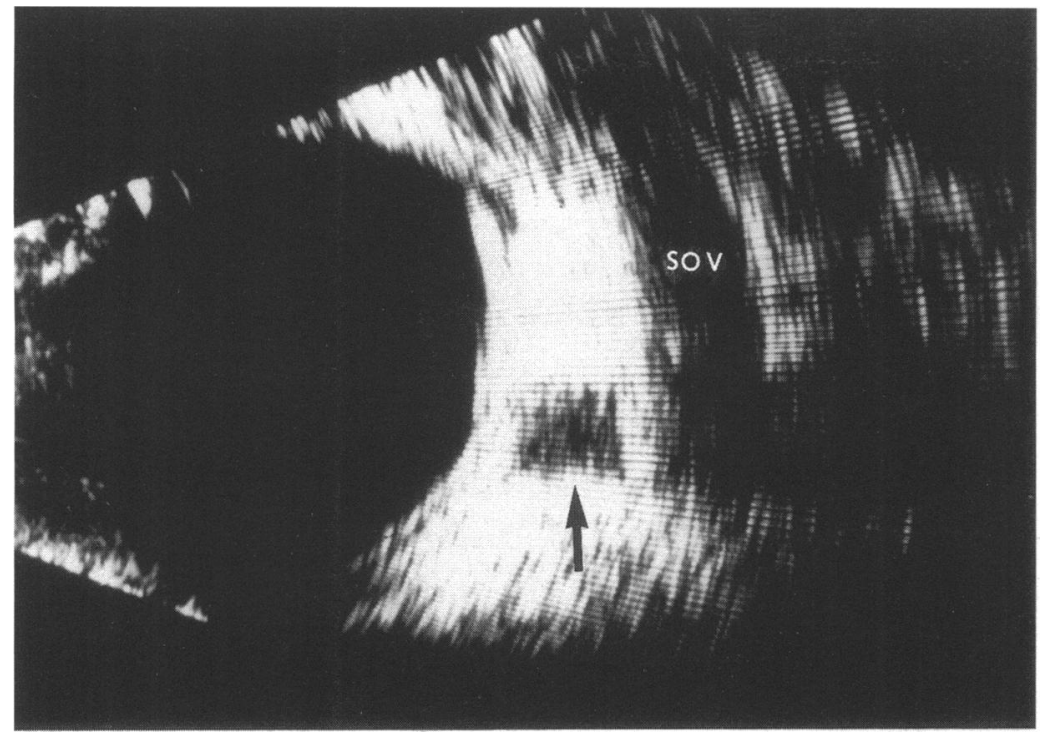

Figure 4 Case 1. Dilated superior ophthalmic vein due to dural-sinus fistula. Vertical transverse B-scan echogram through medial orbit shows cross section of optic nerve (arrow) and very dilated superior ophthalmic vein (SOV). Non-directional Doppler examination indicated only a mildly positive Doppler response due to the slow arterial flow.

typical echographic findings of VSO but, owing to sound attenuation by the orbital fat, may not adequately image the causative apical lesions.

Using standardised echography, we have regularly been able to differentiate between the two main causes of VSO, arteriovenous fistulae and compressive mass lesions. Both of these entities may impede orbital venous outflow and result in a rise in episcleral venous pressure. Previously, the evaluation of patients with a gradual onset of red eye and lid swelling, proptosis, and limitation of ocular movements depended ultimately on CT scan or MRI and angiography. ${ }^{76}$ Measurements of episcleral venous pressure ${ }^{22}$ may be
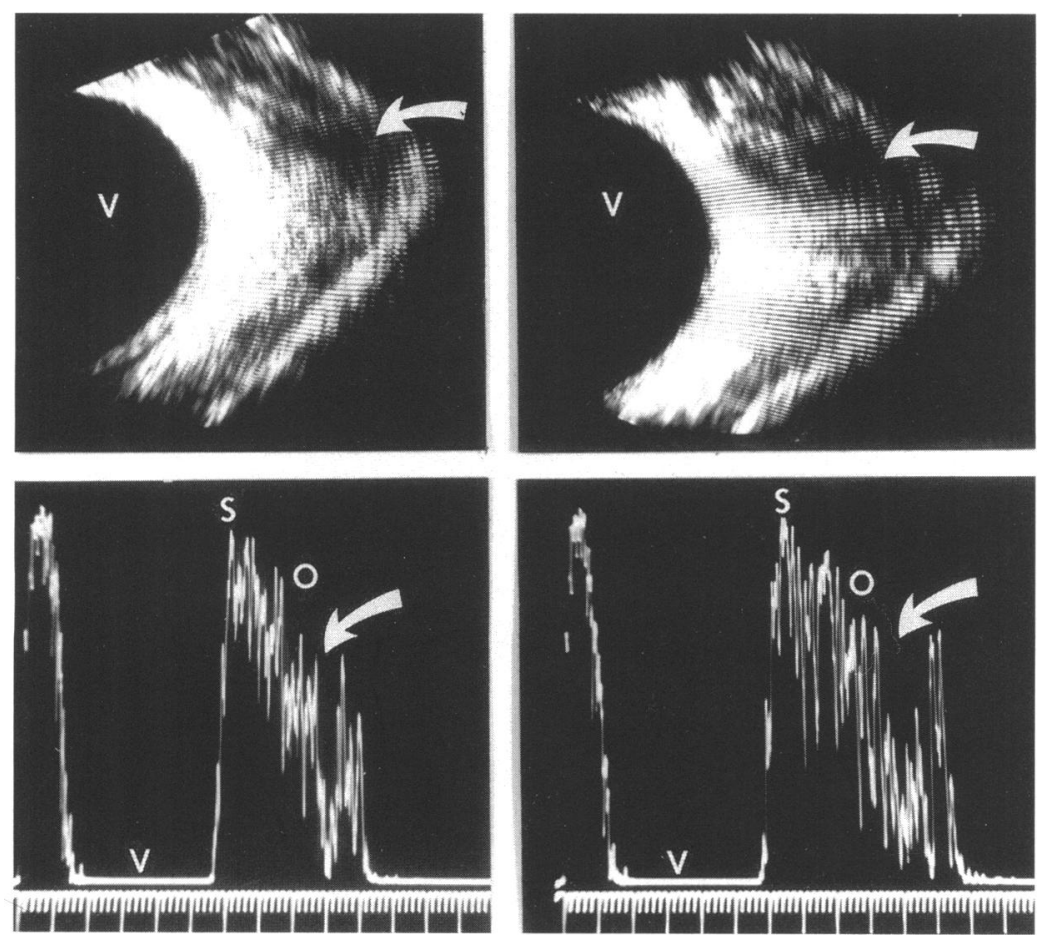

Figure 5 Case 2. B-scan (top) and standardised A-scan (bottom) echograms from normal right orbit (left) and abnormal left orbit (right). V=vitreous cavity. Arrows delineate apex of orbit. Note widened appearance of echograms in the left orbital apex corresponding to inflammation in that area. $S=$ sclera. $O=$ orbital fat.
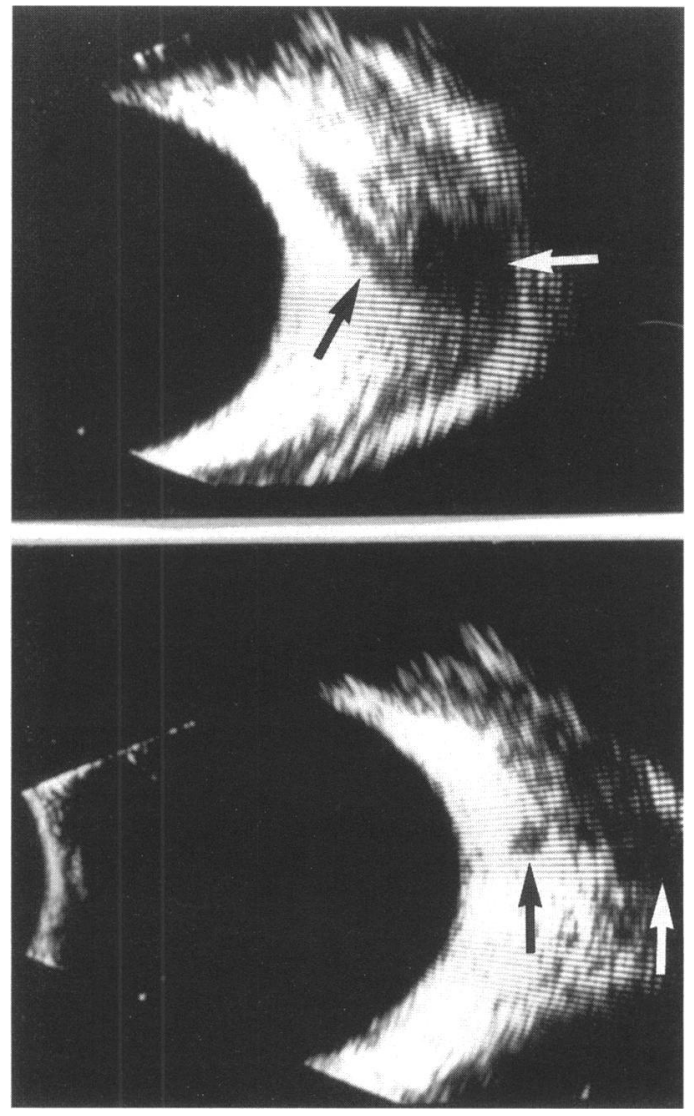

Figure 6 Case 2. B-scan echograms showing mildly dilated superior ophthalmic vein. Top=horizontal transverse scan shows homogeneous lesion posteriorly (white arrow) and slightly dilated superior ophthalmic vein (black arrow). Bottom=longitudinal scan shows dilated vein in cross section (black arrow) and lesion in long section (white arrow).

performed to evaluate venous outflow obstruction, but the precise cause of such venous obstruction is not readily identified. The present study demonstrates that VSO related to arteriovenous fistula results in a greater increase in cumulative muscle thickness and a greater absolute uniocular intraocular pressure, compared with the nonvascular group. We found that a greater uniocular absolute IOP occurred even in the nonfistula group of disorders we studied. This feature has not been documented previously in the literature. VSO should be considered in the context of uniocular pressure increases, particularly when accompanied by an ocular bruit. However, ocular bruits were perhaps a surprisingly uncommon finding in patients with arteriovenous fistulae. Echographically, VSO should be suspected when the orbitopathy is not due to an orbital mass lesion, Graves' disease, or orbital myositis, and when enlarged extraocular muscles are accompanied by distended orbital veins and optic nerve sheath enlargement.

The fact that both the fistulae and the more heterogeneous non-fistula group shared similar echographic and clinical features, including the common denominator of orbital venous congestion, may provide some insight into the pathophysiological mechanisms responsible for the production of orbital symptoms and signs. Furthermore, echography is a non-invasive, safe, cost effective, 


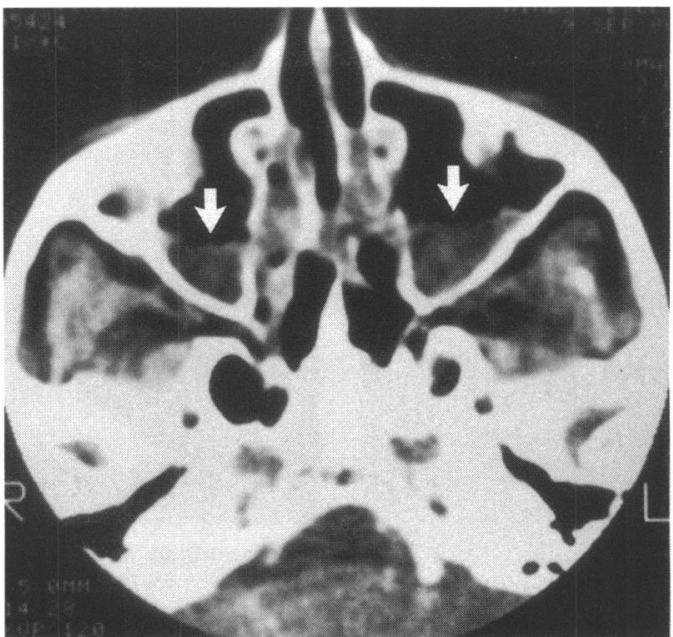

Fig $7 A$

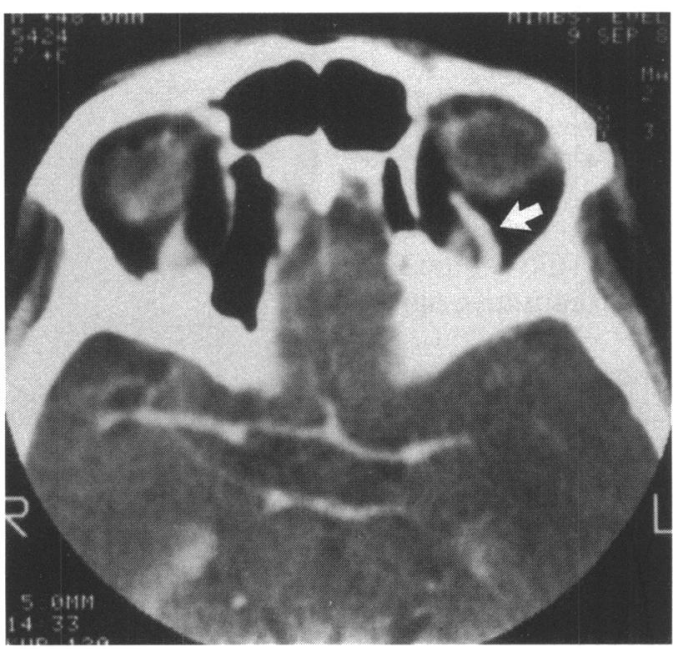

Fig $7 B$

Figure 7 Case 2. Computed tomography scans. (A) Coronal section shows fluid level in maxillary sinuses (arrows). (B) Axial section shows moderate dilatation of superior ophthalmic vein (arrow) in left orbit, and increased density lesion in orbital apex.

and reproducible method that can differentiate not only the causes of enlarged extraocular muscles but also serve as an invaluable aid in the differential diagnosis of the syndrome of venous stasis orbitopathy.

1 Ossoinig KC. Standardized echography: basic principles, clinical applications and results. Int Ophthalmol Clin 1979; 19: $127-210$.

2 Ossoinig KC. Echography of the eye, orbit and periorbital region. In: Arger PH, ed. Roentgenology. New York: Wiley, region. In: Arger

3 Byrne SF. Standardized echography of the eye and orbit. Neuroradiology 1986; 28: 618 .

4 Atta HR. Techniques and applications of diagnostic ultrasound. In: Easty DL, ed. Current ophthalmic surgery. London: Baillière Tindall, 1990, 31-46.

5 Byrne SF, Glaser JS. Orbital tissue differentiation with standardized echography. Ophthalmology 1983; 90 1071-90.

6 Hodes BL, Wienberg P. A combined approach for the diagnosis of orbital disease. Arch Ophthalmol 1977; 95: 781-8.

7 Dick AD, Nangia V, Atta HR. Standardized echography in the differential diagnosis of extraocular muscle enlargethe differential diagnosis of

8 Dick AD, Atta HR, Forrester JV. Lithium induced orbitopathy. Arch Ophthalmol 1992; 110: 452-3.

9 Atta HR, NcCreath G, McKillop JH, Forrester JV Thompson $\mathrm{R}$, Wilson $\mathrm{R}$, et al. Ophthalmopathy in early thyrotoxicosis - relationship to thyroid receptor antibodie and effects of treatment. Scott Med F 1990; 35: 41-4.

10 Ossoinig KC. Ultrasonic diagnosis of Graves' disease. In: Gorman CA, ed. The eye and orbit in thyroid disease. New York: Raven Press, 1984: 185-211.

11 Wan WL, Cano MR, Green RL. Orbital myositis involving the oblique muscles. An echographic study. the oblique muscles. An

12 Ossoinig KC, Hermsen VM. Myositis of extraocular muscles diagnosed with standardised echography. In Hillman JS, Lemay MM, eds. Ophthalmic ultrasonography. The Hague: Dr W Junk, 1983: 381-92.

13 Byrne SF, Green RL. Ultrasound of the eye and orbit. London: Mosby Year Book, 1993.

14 Phelps CD, Thompson HS, Ossoinig KC. The diagnosis and prognosis of atypical carotid-cavernous fistula. $A m$ Ophthalmol 1982; 93: 423.

15 Watson NJ, Dick AD, Huchinson CC. A case of sinusitis presenting with sphenocavernous syndrome: discussion of presenting with sphenocavernous syndrome: discussion of 16 Keltner JL. A red eye and high intraocular pressure. Surv Ophthalmol 1986; 31: 328.

17 Byrne SF, Gendron K, Glaser JS, Feuer W, Atta HR Diameter of normal extraocular recti muscles with echography. Am F Ophthalmol 1991; 112: 706-13.

18 Byrne SF. Evaluation of the optic nerve with standardised echography. In: Smith LJ, ed. Neuro-ophthalmology. New York: Field, Raicha and Associates, 1986: 45-66.

19 Atta H. Imaging of the optic nerve with standardised echography. Eye 1988; 2: 358-66.

20 Ossoinig KC. Standardised echography of the optic nerve. In Till P, ed. Ophthalmic echography. Proceedings of the 13th Til P, ed. Ophthalmic echography. Proceedings of the 13th

21 Ossoinig KC. Superior ophthalmic vein thrombosis - an echographic diagnosis. In: Ossoinig KC, ed. Ophthalmic echography. Proceedings of the 10th SIDUO congress. Amsterdam: Kluwer Academir Publishers, 1987.

22 Phelps CD, Armaly MF. Measurement of episcleral venous pressure. Am F Ophthalmol 1978; 85: 35. 